WellBeing International

WBI Studies Repository

1990

\title{
Alternatives to and Reduction of Animal Use in Biomedical Research, Education and Testing
}

John M. Frazier

Johns Hopkins University

Alan M. Goldberg

Johns Hopkins University

Follow this and additional works at: https://www.wellbeingintlstudiesrepository.org/acwp_arte

Part of the Bioethics and Medical Ethics Commons, Laboratory and Basic Science Research Commons, and the Research Methods in Life Sciences Commons

\section{Recommended Citation}

Frazier, J. M., \& Goldberg, A. M. (1990). Alternatives to and reduction of animal use in biomedical research, education and testing. Alternatives to laboratory animals: ATLA.

This material is brought to you for free and open access by WellBeing International. It has been accepted for inclusion by an authorized administrator of the WBI Studies Repository. For more information, please contact wbisr-info@wellbeingintl.org.

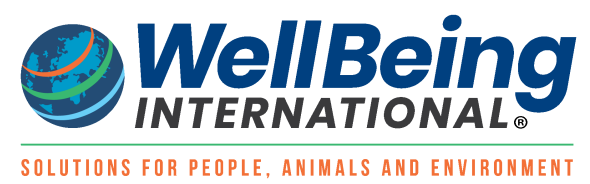




\title{
Alternatives to and Reduction of Animal Use in Biomedical Research, Education and Testing
}

John M. Frazier and Alan M. Goldberg

Johns Hopkins University

\section{$\underline{\text { KEYWORDS }}$}

animal experimentation, animal testing, alternatives

\begin{abstract}
Biomedical endeavours can be divided into three major categories: research, education, and testing. Within the context of each of these categories, activities involving whole animals have made major contributions and will continue to do so in the future. However, with technological developments in the areas of biotechnology and computers, new methods are already reducing the use of whole animals in certain areas. This article discusses the general tissues of alternatives and then focuses on the development of new approaches to toxicity testing.
\end{abstract}

\section{Introduction}

In recent years, societal concerns over the use of animals in various biomedical activities have significantly increased. The ethical issues involved are not new; however, increased awareness of these issues in the scientific community has led to an increased emphasis on the development of new approaches to conducting these traditional activities (1). In the simplest analysis, biomedical activities can be classified into three categories: research, education, and testing. Biomedical research involves the discovery of new knowledge relating to the nature of disease processes and their treatment. These activities have traditionally used animals as models to develop an understanding of the human condition. Despite limitations when using animal models, few question that this approach has been highly successful. Education involves the process of information transfer and can be subdivided into the transfer of factual knowledge and the transfer of skills. Again, animals have been used in activities ranging from teaching students basic principles of physiology to training future surgeons in sophisticated surgical procedures. Testing is a focused activity designed to evaluate the hazards (commercial chemicals) or safety (pharmaceuticals) involved in the use of new chemicals or formulations for beneficial purposes. Over the past 60 years, testing strategies based on whole animal procedures have evolved to provide the experimental database for safety/hazard evaluation. In all three categories, historical precedents have been established that focus on animal models as the foundation for biomedical progress. The important 
question today is whether alternatives exist that can satisfactorily substitute for the currently employed whole animal models in the various biomedical activities.

The likelihood of implementing alternatives in each of the areas of biomedical application -- research, education, and testing -- differs from one area to the next. With respect to refinement and reduction alternatives either are under evaluation or, in many cases, have already been implemented in all areas of biomedical activities. However, when one discusses the possibility of alternatives as full replacements for ongoing approaches, it is necessary to consider each application separately. Replacement alternatives have already been implemented in many areas of education, based on computer and simulation technologies. However, in the area of skilled training (surgical training), it is difficult to envisage the possibility of total replacement. Much can be .done to decrease the use of animals, and endeavours to reach this goal should be encouraged.

For bio-medical research, the concept of replacement is also difficult for most to perceive. Research science is driven by the questions that arise out of clinical experience, laboratory investigation, or human accidents. The purpose of research--is the discovery of new knowledge; and understanding specific biological systems most likely necessitates the use of - whole-animal models. Many biomedical research approaches are currently based on in vitro systems that, in each particular case, represent the best scientific approach. The interaction between whole animals and in vitro models in research is not an either/or situation, but a matter of degree.

In the area of testing, which has received considerable attention in recent years, much progress has already been made in the implementation and development of alternatives. Whether it is possible to fully replace whole animal use is unclear, although animal use can be greatly reduced. The remainder of this article will explore in more detail the issue of testing.

\section{Toxicity Testing}

Industrial technology of the 20th century is capable of producing new chemicals at a rate of several thousand new chemicals and formulations each year. It is essential that any detrimental side-effects that may be associated with the introduction of these new chemicals be identified and evaluated in a timely and efficient manner. In addition, adverse effects resulting from exposure in the occupational environment or accidental exposure of non-target organisms must be elucidated and appropriate remedial procedures established.

Hazard identification, exposure estimation, and the exposure-response relationship are the three major activities of risk assessment. Hazard identification is the process by which potential toxicological problems resulting from the manufacture and use of chemicals are elucidated. Exposure estimation gives some indication of how many people will be exposed to what concentrations, for how long, and under what conditions (route of exposure). For a chemical agent to be of toxicological concern, a reasonable likelihood of human exposure to the agent, in quantities sufficient to produce adverse biological effects must exist. The third activity associated with the risk assessment process, the development of exposureresponse relationships, provides a quantitative relationship of the chance for developing a particular adverse response as a function of exposure to the chemical. Presumably, one such relationship exists for each possible adverse outcome (cancer, kidney damage, reproductive effects, etc.). Exposure-response relationships depend on many factors that affect the intrinsic toxicity of a chemical, such as route of exposure (ingestion, inhalation, dermal contact) and host characteristics (age, nutritional status, genetic background, among others). Combining exposure and the exposure-response relationship provides a quantitative estimate of the potential toxicological risk of a given chemical to a given population under the conditions of proposed use. 
Within the context of risk assessment, the main objectives of toxicity testing are: a) to determine which potential adverse effects are of concern for a given chemical (hazard identification); and $b$ ) to provide adequate data to estimate the quantitative exposure-response relationship in the animal of concern.

Traditionally, these goals were attained through the use of whole-animal studies. Early toxicity testing in the 1920s and 1930s focused mainly on evaluating acute lethality. The LD50 test was developed to provide a statistically-defined estimate of the dose of a chemical that produced $50 \%$ mortality in a population of animals. Later, in the 1940s, ocular irritation testing was standardised by the Draize protocol (2). Over the years, other procedures were developed to evaluate various aspects of toxicological concern.

In vitro systems have been traditionally employed in biomedical research. In the 18th and 19th centuries, classic in vitro experiments on muscle physiology were conducted with isolated muscle preparations. Since then, many other in vitro systems have been developed to investigate physiological and biochemical processes (3). Only recently have in vitro systems been utilised in the area of toxicity testing (4). Carcinogen testing initially was the major focus of in vitro toxicity testing, the result of pioneering work by Ames. et al. $(5,6)$ on Salmonella-based mutagenicity test systems in the late 1960 s and 1970 s. More recently, the use of in vitro systems has been advocated for evaluating toxicological responses in areas other than carcinogenic risk. In the mid-1970s, interest in in vitro alternatives to in vivo teratogenicity testing developed. Later, the areas of acute toxicity, particularly the LD50 (7, 8), and ocular irritation, received major attention. However, in a review of ocular irritation testing by the US Environmental Protection Agency in 1981, only five in vitro test systems were identified (9).

Since 1980, rapid developments have occurred in the field of in vitro toxicity testing. Initially, these efforts were promoted by individuals $(3,7,8,10)$. However, early on, several organisations developed to pursue these activities. In the United States, the first major programme to focus on alternatives to Draize ocular irritation testing was initiated at Rockefeller University under the direction of Dr Dennis Stark, and supported by the Revlon Corporation. Soon afterwards, the Cosmetic, Toiletry and Fragrance Association provided support to establish the Johns Hopkins Center for Alternatives to Animal Testing (CAAT), under the direction of Dr Alan Goldberg. Both of these organisations have fostered the development of in vitro toxicity testing in the United States. In Europe, the major organisations involved in alternative testing issues are the Fund for the Replacement of Animals in Medical Experiments (FRAME), coordinated by Dr Michael Balls of the University of Nottingham, UK, and the European Research Group for Alternatives in Toxicity Testing (ERGATT). Together, these four organisations have had a major impact on in vitro toxicity testing outside the realm of carcinogenicity testing. Recently, the Japanese Society for Alternatives to Animal Experiments was established.

Technological developments in the areas of cell culture and bioanalytical techniques have opened new avenues for in vitro toxicity testing. New approaches to culturing important cell types led to the development of new test systems. Developments in bioanalytical techniques, such as monoclonal antibodies that allow for the analysis of marker proteins as test endpoints, also contribute to the development of new tests. Combining new cell cultures with new ways to measure toxicologicla endpoints stimulates advances in in vitro toxicity testing technology.

\section{Classification of Testing Objectives}

Before discussing specific testing activities, it is useful to have some perspective on the general objectives of these activities. Toxicity testing can be performed at various levels with respect to the toxicological information derived (Figure 1). Listed in increasing order of information content, terms used to describe these levels are toxic potential, potency, hazard assessment, and risk assessment. 
Assessment of toxic potential determines the mere possibility that a chemical can produce an adverse effect independent of its likelihood or its severity under expected conditions of exposure. Potency evaluations provide the next highest level of information; determination of potency establishes the relative toxicity of a chemical and can be used to rank or classify chemicals. Potency measurements, although often quantitative in nature, depend on the test system used for the assessment and therefore do not provide absolute measures of toxicity. Hazard assessment, on the other hand, is quantitative and provides specific information on the likelihood of particular adverse effects in the animal of interest. Finally, risk assessment, the highest level of information, specifies the probability of an adverse effect occurring within a population given a particular likelihood of exposure. Particular toxicity testing activities can be focused at any one of these levels of toxicity evaluation.

Figure 1: Classification of in vitro toxicity testing by level of testing and type of testing

\begin{tabular}{|l|l|l|l|}
\cline { 2 - 5 } \multicolumn{2}{|c|}{} & \multicolumn{2}{|c|}{ TYPE OF TESTING } \\
\hline LEVEL OF TESTING & \multicolumn{2}{|c|}{ SCREENING } & \multicolumn{2}{|c|}{ ADJUNCT } & \multicolumn{2}{c|}{ REPLACEMENT } \\
\hline POTENTIAL ASSESSMENT & & & \\
\hline POTENCY ASSESSMENT & & & \\
\hline HAZARD ASSESSMENT & & & \\
\hline RISK ASSESSMENT & & & \\
\hline
\end{tabular}

Level of testing is listed in increasing order of information content. Shaded boxes indicate areas where in vitro testing is currently being applied, and open boxes show areas in which in vitro testing is under development. (See text for definition of terms).

From a different perspective, the objective of a testing activity can be categorised by the type of testing: screening, as adjuncts, or as replacements. Screening tests are preliminary activities expected to be followed by more definitive testing. Screening can be used to set priorities; for example, which chemicals should be retained in development programme based on the likelihood of lower toxicity. The screening test does not make a final determination on toxicity, but is used as a component of the decision-making 
process in product development. A second type of testing is as adjuncts. Here the testing information is more definitive than it is in screening, but it is not sufficient in and of itself for making final regulatory decisions. Several categories of testing activities fall into this area. One is mechanistic studies in in vitro systems that help resolve discrepancies in animal test data; another is tests used as early tiers in a tier testing strategy for toxicity classification. Another area where in vitro tests can serve as adjuncts is for evaluation of the intrinsic cellular toxicity of chemicals. By using a battery of in vitro systems to develop basic information about the mechanism of action of a chemical, important toxicological information can be developed to aid in the final safety evaluation. Finally, in vitro tests have been proposed as replacements for existing in vivo testing procedures. The replacement of classic whole-animal testing activities is not yet possible and will require significant scientific developments, particularly in the area of in vitro/in vivo extrapolation, before it becomes a reality.

\section{In Vitro Tests}

In vitro tests are rapidly being developed as alternatives to using whole animals in various areas of toxicity testing. Research activities can be classified into at least seven categories: cytotoxicity; inflammation and irritation; genotoxicity; teratogenicity; target organ toxicity; toxicokinetics; and structureactivity relationships. Although not necessarily an inclusive listing, these categories cover the most general areas in which in vitro approaches are actively being investigated.

\section{Cytotoxicity}

In vitro cytotoxicity assays have a long history, arid many have been developed over the years $(4,11)$. Some were developed for special purposes, such as screening potential antineoplastic drugs for their ability to kill cancer cells, while others were developed for more general purposes. Cytotoxicity can be evaluated with any cell type that can be cultured in vitro, and the number of methods for evaluating whether cells are viable has increased in recent years. Thus, a multiplicity of test systems are available. Two cytotoxicity test systems that have received particular' attention are the assay developed by FRAME (12), and the neutral red uptake assay developed by the research group at Rockefeller University (13).

\section{Inflammation and irritation}

Research in this area involves eye and skin irritation problems. Most toxicologists are familiar with the Draize eye test, but alternative approaches to ocular irritation testing are clearly needed. Significant research effort has focused on developing in vitro methods to provide information equivalent to that obtained from either the Draize eye or skin test. The Johns Hopkins CAAT identified 34 potential in vitro tests that can contribute to ocular irritation testing (14).

\section{Genotoxicity}

Many approaches to mutagenicity and carcinogenicity testing, beginning with the Ames assay, have been proposed or are under development (15). Genotoxicity has probably been the most adequately funded and active area of in vitro approaches to toxicity testing, because conducting whole-animal tests for carcinogenicity is extremely expensive and time-consuming. Therefore, significant economic incentives exist for developing alternatives to in vivo carcinogenicity testing.

\section{Teratogenicity testing}

Developmental abnormalities resulting from chemical agents are another important area of toxicological concern. The story of thalidomide is a constant reminder of the toxicological problems associated with fetal development, and is frequently used by both animal rights activists and the scientific community to 
prove the value, or lack thereof, of animal testing. The activists indicate that not all species are affected by thalidomide. Although this is true, it was the collective animal studies that provided the evidence that thalidomide is a teratogen. In vitro and whole-animal metabolic studies later demonstrated the mechanism of thalidomide-induced teratogenesis. In vitro approaches to this area of toxicity testing are being actively pursued (16).

\section{Target organ toxicity}

Research efforts focus on cell cultures from specific organs to evaluate selective toxicity that occurs in those particular organs. Extensive in vitro research is conducted on mechanisms of action. Progress has been made in many areas, including hepatotoxicity $(17)$, nephrotoxicity $(18)$ and neurotoxicity $(19,20)$.

\section{Toxicokinetic testing}

Understanding the distribution and metabolism of toxicants is essential for toxicological risk assessment (23). Many studies have investigated the relationship between in vitro and in vivo toxicokinetics. However, because much remains to be accomplished, this area should receive priority in the future.

\section{Structure-activity relationships}

Current efforts attempt to correlate some general toxicological responses (lethality, ocular irritation, cytotoxicity) with chemical parameters computed from the molecular structure of the test chemical (22, 23). At present, these methods are highly empirical, but are of value for regulatory purposes when other data are not available. More mechanistic approaches are being developed (24). In the future, when specific mechanistic effects can be related to chemical structure, these techniques may provide the ultimate alternative method, since they depend only on computer analysis of the physicochemical structure of molecules.

No single in vitro test will answer all toxicological questions. The many areas of toxicological concern noted above involve very different biochemical-mechanistic processes; consequently, no one test is currently adequate to evaluate this broad spectrum of endpoints. It will take batteries of in vitro tests to obtain the information necessary to evaluate human risk resulting from exposure to potentially toxic chemicals.

An important advantage of in vitro techniques in toxicity testing is that human cells can be used (27). Advances in the biotechnology of human cell culture, in the cryopreservation of human tissue, and in genetic engineering techniques to provide continuous supplies of normal, differentiated human cells are helping to solve the problems of interspecies extrapolation of test data and to reduce the need for animals in providing cells and tissues for toxicity testing systems.

\section{Validation}

Before any new test can be accepted, it must be validated. This process, which establishes the credibility of a method for a particular purpose, consists of: a) establishment of test reliability; and b) development of the scientific basis for interpretation of test results. A strategy for approaching validation involves a series of logical steps $(26,27)$. First, the research scientist who actually developed the original test (called the primary laboratory) must conduct an "intralaboratory assessment" and satisfy two requirements. One requirement is standardising the test, meaning that the test protocol must be defined in a form that can be transferred to other laboratories (referred to as secondary laboratories) with a reasonable likelihood of success. The second requirement is that the primary laboratory must conduct a blind study to establish the feasibility of the test as a useful alternative for some specific purpose and to provide quality-control 
data for later comparisons. Once standardised in the primary laboratory, the test must be introduced into the real world to see if it will work. This process, referred to as "interlaboratory assessment", is used to evaluate whether secondary laboratories can obtain reproducible results. If secondary laboratories can replicate results obtained in the primary laboratory, it is possible to start building a broad database ("test database development") that can be used to determine how reliable test results are by evaluating the false-positive rate, false-negative rate, sensitivity, specificity, and predictive value of the test: These data are necessary before the test can be taken to the final level of development. The final stage of validation is the time-consuming process of developing an understanding of the scientific basis of the test and generating a large database for interpretation. The main problem in the implementation of in vitro testing today is that the scientific community does not have the 50-60 years of experience that it has with in vivo testing. It will take time to overcome the problems inherent in introducing new technologies and having them accepted. Cumulative experience is what will ultimately boost in vitro approaches over the validation hurdles.

Table 1: Tier testing strategy for incorporating in vitro toxicity testing into the risk assessment process

\begin{tabular}{|cll|}
\hline Tier & Title & Approach \\
\hline 1 & Structure-activity analysis & $\begin{array}{l}\text { Search literature for toxicity of structurally related chemicals based on } \\
\text { physicochemical properties. }\end{array}$ \\
2 & In vitro testing as screens & $\begin{array}{l}\text { Test for various aspects of toxicity using in vitro testing systems. Use } \\
\text { information provided to make initial regulatory decisions. }\end{array}$ \\
3 & In vivo testing & $\begin{array}{l}\text { Test chemicals in vivo only when additional toxicologic information is needed. } \\
\text { Note that little or no toxicity is expected at this stage because the more toxic } \\
\text { chemicals will have been identified at earlier stages. }\end{array}$ \\
\hline
\end{tabular}

\section{Incorporation of In Vitro Methods in Risk Assessment}

In vitro toxicity testing is a new, branch of toxicology and, as such, will require extensive research and development to gain acceptance as a credible methodology for the toxicological evaluation of new chemicals. In the short run, in vitro toxicity testing can be incorporated into the risk assessment process through the use of tier testing strategies (Table 1). At an early level in a tier testing strategy, in vitro methods can be used to select chemicals with the lowest probability for toxicity. Using this approach, only chemicals expected to have little or no toxicity are ever tested in live animals. Such a strategy can be utilised early in the development of new products to save the time and research and development costs that would otherwise be lost, if invested in products that could not pass regulatory safety evaluations prior to marketing. Finally, by looking further into the future, one can foresee a possible risk assessment strategy that will be organised as illustrated in Figure 2. The risk of new chemicals will be evaluated on the basis of in vitro data and structure-activity relationships rather than whole-animal testing.

Interest in the investigation of basic scientific questions that arise when innovative approaches are applied to the problem of toxicity testing and safety evaluation has increased rapidly in the last 15 years. Early questions in the area of carcinogenicity testing have been investigated in academic, government, and industrial laboratories under the auspices of the National Toxicology Program (NTP) in the United States, and the International Agency for Research on Cancer in France. For more than a decade, the NTP has also supported fundamental research into alternatives in the area of teratogenicity. In the 1980s, the Johns Hopkins CAAT has been involved in research, development, and validation activities in all areas of toxicity testing, other than genotoxicity. 
Figure 2: Future strategy for toxicity testing

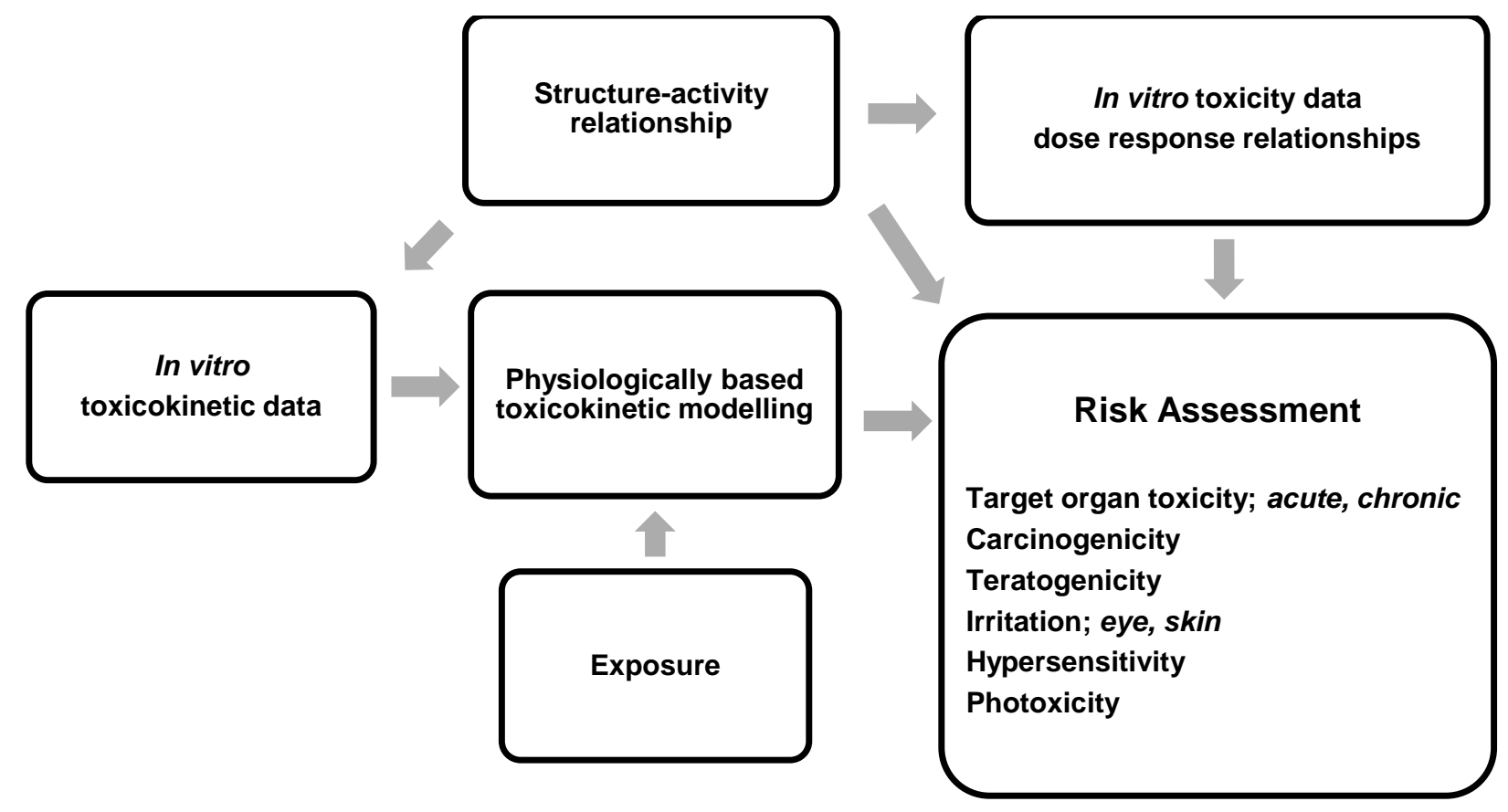

In vitro toxicity and toxicokinetic data will be utilised to provide the experimental database for risk asessment. In vitro toxicokinetics data will be used in computer-generated, physiologically based toxicokinetic models, along with traditional exposure estimates, to predict the concentrations of active forms of the chemical in the critical tissues. In vitro dose-response relationships will be used to predict potential toxicologic risks associated with the expected level of exposure. All these processes will be aided by structure-activity relationships based on mechanical responses. Such a strategy would maximise the use of alternative toxicity testing methods in chemical safety evaluations.

\section{Johns Hopkins Center for Alternatives to Animal Testing}

The Johns Hopkins CAAT was founded in 1981 to address the major issues facing the development of alternatives for toxicity testing. The goal of the Center is to develop and disseminate appropriate scientific knowledge concerning innovative non-whole-animal methods for fully evaluating the safety of commercial and therapeutic products. More specifically, CAAT: a) encourages basic research that will provide the foundation for in vitro or other nonanimal test procedures for examining the toxicity of chemicals and/or commercial products; b) develops specific methodologies that will provide alternative approaches to whole-animal studies for the evaluation of safety; c) disseminates research progress through symposia, publications and workshops; and d) encourages technology transfer and the use of in vitro methods in regulatory consideration and product development.

To attain these goals, the Center has engaged in a variety of activities organised into three major programmes: research; validation and technology; and education and communication.

\section{Research}

The Center's research programme consists of two components: the Research Grants Program and the In Vitro Toxicology Laboratory. The Center has demonstrated that the investigator-initiated, peer-reviewed grant process can produce major advances in a goal-oriented programme. This process has hastened the development of alternatives and has helped to define the new discipline of in vitro toxicology. By the end of fiscal year 1989, CAAT will have dispersed more than $\$ 2$ million for more than 65 research projects. 
Already, the research programme has produced more than 207 articles, book chapters, and symposium presentations, including 85 papers written for peer-reviewed scientific journals.

To supplement its research programme, CAAT has established its own In Vitro Toxicology Laboratory. The laboratory focuses on the development of new in vitro methods for chemical safety evaluation and is involved in training researchers in vitro toxicology.

\section{Validation and technology transfer}

Validation and technology transfer activities of CAAT are conducted through a standing committee. The objective of the Committee on Validation and Technology Transfer is to catalyse the transfer of new testing technology from the research laboratory to practical application in toxicity assessment. This committee is charged with developing a framework to assess existing programmes and coordinate future activities, establishing a scientific structure for validation, and maintaining strong links with regulatory, academic, and industrial activities. Long-range plans include developing a data bank to identify and catalogue validation activities and in vitro methodologies in the United States developing an administrative framework for validation, establishing the criteria for chemical selection for validation, and forming a network of organisations for the exchange of information on validation activities.

Workshops are one mechanism the committees use to promote technology transfer. In 1989, CAAT held the first in a series of technical workshops. The workshop addressed two problem areas: a) identification of artifacts that adversely affect in vitro test results; and b) techniques to handle insoluble test chemicals in in vitro test systems. Representatives from the regulatory and corporate communities participated in a two-day workshop and collaborated in writing a technical report of the workshop. The proceedings of the workshop were published by CAAT (28). Topics for future workshops include chemical selection for validation; interpretation, extrapolation and relevance of in vitro data; good laboratory practices for in vitro testing; and culture conditions and how they affect toxicity testing, validation, and information transfer.

\section{Education and communication}

Through its publications and presentations, CAAT has successfully drawn greater attention to new approaches to toxicity testing and provided essential information to scientists, industry executives, government officials, animal protection advocates, and the news media. The CAAT newsletter (Alternative Methods in Toxicology) has grown from an initial circulation of 1000 to more than 20,000 reaching key international audiences.

Academic, industrial, and governmental scientists view the CAAT symposium as one of the major international meetings on in vitro toxicology. These activities not only serve as a forum for cutting-edge research, but also provide direction and cohesion to the new discipline. Alternative Methods in Toxicology presents the proceedings of the annual symposia, as well as special topics of importance; Volume 7 was published in the fall of 1989.

The CAAT Government Liaison Committee is a mechanism for exchanging information with interested government representatives on animal issues, alternatives research, and regulatory acceptance of tests. Through a regular exchange, CAAT hopes to heighten government interest in alternative research issues and begin a long-term collaborative-education programme leading to regulatory acceptance of alternative testing methodologies. The Committee for Public Information and Resources Development was established to educate the public on the complex issues surrounding animal testing and alternatives.

Through a combination of these programmes, CAAT has been successful in promoting the development of the new science of in vitro toxicity testing, as well as increasing awareness by the general public and 
the scientific community of the scientific issues relating to toxicity testing. It is hoped that through the application of current scientific knowledge and the development of new knowledge through coordinated and focused research, non-whole-animal testing methods can be established as a significant component of the chemical safety evaluation process.

\section{Conclusion}

The use of animals in biomedical activities - research, education, testing -- has come under increasing scrutiny in recent years as a result of various factors, including economics, advances in biotechnology, and animal welfare concerns. In many situations, new approaches allow for refinement, reduction, and, in some cases, replacement of whole animals in activities that traditionally have depended on these model systems. In the area of toxicity testing and safety evaluation, non-whole-animal techniques have made significant contributions as screens and adjuncts. Whether alternative testing methods will completely replace in vivo toxicity testing is a question that only time can answer. In the meantime alternative methods can be employed to improve predictive toxicology and at the same time, significantly reduce pain and distress in animals.

\section{Acknowledgements}

Support for the authors of this manuscript is provided by the Johns Hopkins Center for Alternatives to Animal Testing and by the National Institutes of Health Division of Research Resources (P40 RR0523601). The authors would like to thank Robbie Betts for his secretarial assistance.

\section{References}

1. Alternatives to Animal Use in Research, Testing and Education. (1986). 441 pp. Washington, DC: US Congress, Office of Technology Assessment.

2. Draize, J.H., Woodward, G. \& Calvery, H.O. (1944). Methods for the study of irritation and toxicity of substances applied topically to the skin and mucous membranes. Journal of Experimental Therapeutics 82, 377-390.

3. Rowan, A.N. \& Stratmann, C.J., eds (1980). The Use of Alternatives in Drug Research. Baltimore MD: University Park Press.

4. Goldberg, A.N. \& Frazier, J.M. (1989). Alternatives to animals in toxicity testing. Scientific American 261, 24-30.

5. Ames, B.N. (1971). The detection of chemical mutagens with enteric bacteria. In Chemical Mutagens: Principles and Methods for Their Detection (ed. E.A. Hollaender), pp. 267-282. New York: Plenum Press.

6. Ames, B.N., Duaston, W.E., Yamaaki; E. \& Lee, F.D. (1973). Carcinogens are mutagens: a simple test system combining liver homogenates for activation and bacterial detection. Proceedings of the National Academy of Sciences of the United States of America 70, 2281-2285.

7. Zbinden, G. \& Flury-Roversi, M. (1981). Significance of the LD50 test for the toxicological evaluation of chemical substances. Archives of Toxicology 47, 77-99.

8. Zbinden, G. (1989). Acute toxicity testing: public responsibility and scientific challenge. In Benchmarks: Alternative Methods in Toxicology (ed. M.A. Mehlman), pp. 3-13. Princeton, NJ: Princeton Scientific.

9. Anon. (1981). Eye irritation testing: an assessment of methods and guidelines for testing materials for eye irritancy. Washington, DC: US Environmental Protection Agency.

10. Nardone, R.M. (1983). Neurotoxicity testing: an in vitro strategy. Annals of the New York Academy of Sciences 407, 458-469. 
11. Berky, J. \& Sherrod, C., eds (1977). In Vitro Toxicity Testing, 438 pp. Philadelphia, PA: Franklin Institute Press.

12. Clothier, R.H., Hulme, L., Ahmed, A.B., Reeves, H.L., Smith, M. \& Balls, M.J. (1988). In vitro cytotoxicity of 150 chemicals to 3T3-L1 cells, assessed by the FRAME kenacid blue method ATLA 16, 84-95.

13. Borenfreund, E. \& Puerner, J.A. (1985). Toxicity determined in vitro by morphological alterations and neutral red absorption. Toxicology Letters 24, 119-124.

14. Frazier, J.M., Gad, S.C., Goldberg, A.M. \& Mcqulley, J.P. (1987). In Alternative Methods in Toxicology, Vol. IV, A .Critical Evaluation of Alternatives to Ocular Irritation Testing (ed. A.M. Goldberg). New York: Mary Ann Liebert.

15. Williams, G.M., Dunkel, V.C. \& Ray, V.A., eds (1984). Cellular Systems for Toxicity Testing, 484 pp. New York: New York Academy of Sciences.

16. Kimmel, G.L., Smith, K., Kochlar, D.M. \& Pratt R. (1982). Overview of in vitro teratogenicity testing: aspects of validation and application to screening. Teratogenesis, Carcinogenesis and Mutagenesis 2, 221-229.

17. Rauckman, E.J. \& Padilla, G.M., eds (1987). The Isolated. Hepatocyte: Use in Toxicology and Xenobiotic Biotransformations, 292 pp. New York: Academic Press.

18. Tyson, C.A. \& Stacey, N.H. (1989). In vitro screens from CNS, liver and kidney for systemic toxicity. In Benchmarks:-Alternative Methods in Toxicology (ed. M.A. Mehlman), pp. 111-136. Princeton NJ: Princeton Scientific.

19. Davenport, C.J., Williams, D.A. \& Morgan, K.T. (1989). Neurotoxicology using cell culture. CIIT Activities 9, 1-8.

20. Shahar, A. \& Goldberg, A.M., eds (1987). Model Systems in Neurotoxicology: Alternative Approaches to Animal Testing. New York: Alan R. Liss Inc.

21. Nuclear Regulatory Commission (1982). Pharmacokinetics in Risk Assessment (Drinking Water and Health), Vol. VIII, 488 pp. Washington DC: National Academy Press.

22. ECETOC Monograph No. 8 (1986). Structure Activity Relationships in Toxicology and Ecotoxicology: An Assessment pp. 1-88. Brussels: ECETOC.

23. Enslein, K. (1988). An overview of structure-activity relationships as an alternative to testing in animals for carcinogenicity, mutagenicity, dermal and eye irritation and acute oral toxicity. Toxicology and Industrial Health 4, 479-498.

24. Rosenkranz, H.S. \& Klopman, G. (1989). CASE, the computer-automated structure evaluation system, as an alternative to extensive animal testing. In Benchmarks: Alternative Methods in Toxicology (ed. M.A. Mehlman), pp. 111-136. Princeton, NJ: Princeton Scientific.

25. Frazier, J.M., Tyson, C.A., McCarthy, C. et al. (1989). Contemporary issues in toxicology: potential use of human tissues for toxicity research and testing. Toxicology and Applied Pharmacology 97, 387-397.

26. Balls, B., Blaauboer, B., Brusick, D., Frazier J., Lamb, D., Pemberton, M., Heinhardt, C., Roberfroid, M., Rosenkranz, H., Schmid, B., Spielmann, H., Stammati, A.L. \& Walum, E. (1990). Report and Recommendations of the CAAT/ERGATT workshop on the validation of toxicity testing procedures. ATLA 18, in press.

27. Frazier, J.M. The Scientific Criteria for Validation of In Vitro Toxicity Tests. Paris: OECD Publications, in press.

28. Frazier, J.M. \& Bradlaw, J.A., eds (1989). Technical Problems Associated with In Vitro Toxicity Testing Systems: A Report of the CAAT Technical Workshop of May 17-18, 1989. Baltimore, MD: Johns Hopkins Centre for Alternatives to Animal Testing. 
